Original

Article

\title{
Impact of the 8th Edition of the UICC-TNM Classification on Clinical Stage 0-IA Lung Adenocarcinoma: Does the New Classification Predict Postoperative Prognosis More Precisely than the Previous One?
}

\author{
Liming Wang, MMsc, ${ }^{1,2}$ Masaki Anraku, MD, PhD,${ }^{1}$ Masaaki Sato, MD, PhD, ${ }^{1}$ \\ Jun-ichi Nitadori, MD, PhD, ${ }^{1}$ Kazuhiro Nagayama, MD, PhD ${ }^{1}$ \\ Kentaro Kitano, $\mathrm{MD}, \mathrm{PhD},{ }^{1}$ and Jun Nakajima, $\mathrm{MD}, \mathrm{PhD}^{1}$
}

\begin{abstract}
Purpose: Early lung adenocarcinoma has been more frequently found recently. The 8th edition of the Union for International Cancer Control (UICC)-Tumor Node Metastasis (TNM) classification for lung cancer has been effective since January 2017. This study aims to elucidate advantages of the current classification for patients with clinical stage 0-IA lung adenocarcinoma, in comparison with the older one.

Methods: We retrospectively reviewed the data of clinical stage IA (7th edition) lung adenocarcinoma patients who underwent surgery at our institute from 2001 to 2012, and reclassified them by the 8th edition. Survival analysis was used to evaluate the impact of the two classifications.

Results: In all, 281 cases were eligible. Clinical T-factors (8th) were significant prognostic factors for overall survival $(P=\mathbf{0 . 0 0 1})$, recurrence-free survival $(P<0.001)$, and cancerspecific survival $(P=0.001)$. However, those in the previous edition were not $(P=0.894$, $P=0.144$, and $P=0.822$, respectively).

Conclusion: The 8th edition of the UICC-TNM classification predicts postoperative prognosis more precisely than the 7th one in clinical stage 0-IA lung adenocarcinoma. It is probably because the stage distribution of the population, which included in the research project the 8th edition based on, has been changed, and the new edition develops more accurate staging criteria for ground-glass nodule (GGN).
\end{abstract}

Keywords: lung cancer, adenocarcinoma, neoplasm staging, prognosis

\footnotetext{
${ }^{1}$ Department of Thoracic Surgery, The University of Tokyo Graduate School of Medicine, Tokyo, Japan

${ }^{2}$ Department of Thoracic Surgery, The 1st Affiliated Hospital of China Medical University, Shenyang, China

Received: February 28, 2018; Accepted: May 1, 2018

Corresponding author: Jun Nakajima, MD, PhD. Department of Thoracic Surgery, The University of Tokyo Graduate School of Medicine, 7-3-1 Hongo, Bunkyo-ku, Tokyo 113-8655, Japan

Email: nakajima-tho@h.u-tokyo.ac.jp

(C)2018 The Editorial Committee of Annals of Thoracic and Cardiovascular Surgery. This work is licensed under a Creative Commons Attribution-NonCommercial-NonDerivatives International License.
}

\section{Introduction}

Lung cancer is among the malignant tumors with the high morbidity and mortality in the world now, ${ }^{1)}$ it has a high incidence in East Asia. ${ }^{2)}$ Adenocarcinoma is the majority of pathological types in non-small cell lung cancer (NSCLC). Clinically, besides of improving the diagnosis and treatment of lung cancer, it is also of great importance to predict the prognosis accurately. Union for International Cancer Control (UICC)-Tumor Node Metastasis (TNM) staging system is based on anatomy, 
with an accurate description of the extent of tumor involvement, it closely relates to the prognosis. UICC updates and revises the TNM staging criteria of lung cancer every few years. The previous (7th) edition was published in $2009,{ }^{3)}$ but the number of patients with early lung cancer has been increasing in Asia over the past decade. ${ }^{4)}$ Meanwhile, the diagnosis and treatment of lung cancer have made great progress. As a result, small lung cancers classified as stage IA has been more frequently found recently. So, the 7 th edition cannot meet current clinical needs, especially for patients with early lung cancer. In this situation, UICC published the 8th edition in January 2017.5) The new edition seems to be better in reflecting the diagnosis and treatment of lung cancer globally over the past decade, and may predict the prognosis more accurately. Comparing with the 7th edition, many changes had been made to clinical stage IA, the most important was that the size of the tumor was further subdivided. Meanwhile, "stage 0" was introduced in the 8th edition. To verify whether the new edition is better than the previous one in predicting the postoperative prognosis of the patients with clinical stage 0-IA lung adenocarcinoma, we carried out this study. In the 8th classification, the whole size of ground-glass nodule (GGN) on computed tomography (CT) is neglected for determining T-factor. ${ }^{5)}$ However, the 8th classification is based on a worldwide database in which the size of the tumor was not defined as that of solid part. Therefore, in this study, we also aim to elucidate the accuracy of the new measuring method adopted in the new classification for patients with adenocarcinoma because its size of whole tumor does not always match that of solid tumor.

\section{Materials and Methods}

\section{Study design and patient eligibility}

This is a retrospective and observational study of patients with clinical stage IA (according to the 7th UICCTNM classification) lung adenocarcinoma who had undergone surgical therapy at our institute from April 2001 to December 2012. The protocol of the study was comprehensively approved by the Ethics Committee of The University of Tokyo Graduate School of Medicine (Approval Number: 2406-(5)). The need for patient consent was waived, and all patients gave their informed consent prior to surgery. We assessed the data by CT images and classified them according to the 7th and 8th UICC-TNM classifications. Finally, we got the data which consisted of the clinical T-factors of both of the two classifications.
On the basis of the GGN's appearance on the highresolution computed tomography (HRCT) scans, we classified each GGN as to its type: solid nodule, part solid GGN, or pure GGN. Solid nodule was defined when a GGN completely obscured the entire lung parenchyma within it; and a GGN was classified as pure GGN if none of the lung parenchyma within it was totally obscured; other situations between them will be classified as part solid GGN.6)

\section{Statistical methods}

We used the survival analysis to evaluate the impact of the clinical T-factors of both the 7th and 8th editions on postoperative prognosis. We also analyzed other potential prognostic factors such as age, gender, smoking, obstructive/restrictive disorder, surgical procedure, and vessel/ pleural invasion at the same time. We got the conclusion by comparing the survival curves of the clinical T-factors between the 7th and 8th UICC-TNM classifications.

The primary outcomes, overall survival, is defined as the time from operation until death by any cause, with living patients censored on the date of last follow-up. ${ }^{7)}$ Our secondary outcomes contain recurrence-free survival and cancer-specific survival. Recurrence-free survival is defined as time from operation until first recurrence or death by any cause, whichever happened first, and patients alive without recurrence were censored on the date of last follow-up. ${ }^{8)}$ And cancer-specific survival is defined as the time from operation until death caused by lung cancer, censored survival values represented patients still alive at the time of last follow-up or those patients who died without evidence of lung cancer. ${ }^{9)}$

The data were analyzed using SPSS (Version 16.0). The count variables were categorized into binary or polynary variables according to their properties. As for the continuous variables, set the median as the cutoff value, and then transformed them into binary variables. The survival curves were estimated according to the Kaplan-Meier's method, and the differences between groups were compared using a log-rank test. A P value of less than 0.05 was considered statistically significant.

\section{Results}

\section{Patient characteristics}

In all, 300 cases were registered at the beginning. Preoperative HRCT and postoperative tumor-free surgical margin were required (12 cases were excluded), we also excluded seven cases who were lost during follow-up. 
Table 1 Patient characteristics: continuous variables

\begin{tabular}{lccc}
\hline Variables & Range & Mean \pm SD & Median \\
\hline Age (years) & $34-86$ & $66.0 \pm 9.9$ & 67 \\
Overall survival time (months) & $1-190$ & $79.4 \pm 37.6$ & 79 \\
Recurrence-free survival time (months) & $1-178$ & $72.6 \pm 38.1$ & 73 \\
Cancer-specific survival time (months) & $1-190$ & $79.4 \pm 37.6$ & 79 \\
Whole size of tumor on CT (cm) & $0.6-3.0$ & $1.7 \pm 0.6$ & 1.6 \\
Solid size of tumor on CT (cm) & $0.0-3.0$ & $1.0 \pm 0.8$ & 0.8 \\
\hline
\end{tabular}

CT: computed tomography; SD: standard deviation

Table 2 Relationship between the clinical T-factors of the 7th and 8th editions

\begin{tabular}{rrrrrrrr}
\hline & & \multicolumn{7}{c}{ 8th edition } \\
\cline { 3 - 8 } & & cTis & cT1mi & cT1a & cT1b & cT1c & Sum \\
\hline \multirow{3}{*}{ 7th edition } & cT1a & 59 & 29 & 53 & 78 & 0 & 219 \\
& cT1b & 3 & 4 & 15 & 17 & 23 & 62 \\
& Sum & 62 & 33 & 68 & 95 & 23 & 281 \\
\hline
\end{tabular}

Finally, 281 cases were eligible for the study. All data were evaluated by August 31, 2017. The most recent follow-up date was ranging from July 29, 2001 to August 3,2017 . The mean \pm SD and median of follow-up time were $79.4 \pm 37.6$ months and 79 months, respectively. There were 52 cases with recurrence of lung cancer. In all, 232 cases were alive on their last follow-up date, and 49 cases had been dead. Among these 49 cases, 23 died of recurrence of lung cancer, 5 died of other cancers, 11 died of non-cancer diseases, and 10 were not quite clear. There were 62 cases of pure GGN, 109 cases of part solid GGN, and 110 cases of solid nodule.

The continuous variables of patient characteristics are shown in Table 1. The relationship between the clinical T-factors of the 7th and 8th UICC-TNM classifications is shown in Table 2. Other characteristics are shown in Table 3.

\section{Comparison of the clinical T-factors}

Table 3 shows the three kinds of cumulative 5-year survival rate of each potential prognostic factors according to the relative outcomes, it also presents whether these factors are statistically significant on affecting the postoperative prognosis or not. From the results of the survival analysis, the factors such as age, gender, vessel invasion, pleural invasion, and the clinical T-factor (8th) affected all of the three survivals. On the contrary, surgical procedure and the clinical T-factor (7th) had relationship with none of the three survivals.

Figure 1 shows the survival curves of patients stratified with clinical T-factors (8th) were more clearly separated than that of the 7 th: $\mathrm{P}$ values of the difference in overall survival, recurrence-free survival, and cancerspecific survival rates were $0.894,0.144$, and 0.822 in the 7 th edition, and $0.001,<0.001$, and 0.001 in the 8 th edition, respectively.

\section{Discussion}

Before Jang et al. ${ }^{10)}$ found that the ground-glass opacity (GGO) on thin-section CT was also possible to be the bronchioloalveolar carcinoma (BAC), it was considered to be the pneumonia, pulmonary fibrosis, or pulmonary hemorrhage habitually. Then, many studies confirmed that the BAC component of adenocarcinomas corresponded to the GGO portion of the nodules. ${ }^{11,12)}$ The pathological characteristic of $\mathrm{BAC}$ is a kind of lepidic growth pattern, in which the cells proliferate along the alveolar walls, ${ }^{13)}$ and it keeps with the GGO characteristics on CT perfectly. Then since the 2015 World Health Organization (WHO) pathological classification, BAC has been replaced by "adenocarcinoma with lepidic growth." ${ }^{14)}$ And now the diagnosis of lesions which showing the GGN features on CT varies widely, it includes the benign ones like inflammation, the precancerous ones like atypical adenomatous hyperplasia (AAH), and the malignant ones like adenocarcinoma. Recently, the frequency of identification of small lung adenocarcinomas, especially the GGN ones, has been increased since the improvement of CT and the ascension of CT scanning for early lung tumor in the health examinations without symptoms. ${ }^{15,16)}$ Such small lung adenocarcinomas often contain GGO components as visualized on HRCT.

There were no measurement standards for GGN lung adenocarcinomas before and within the 7th edition of 


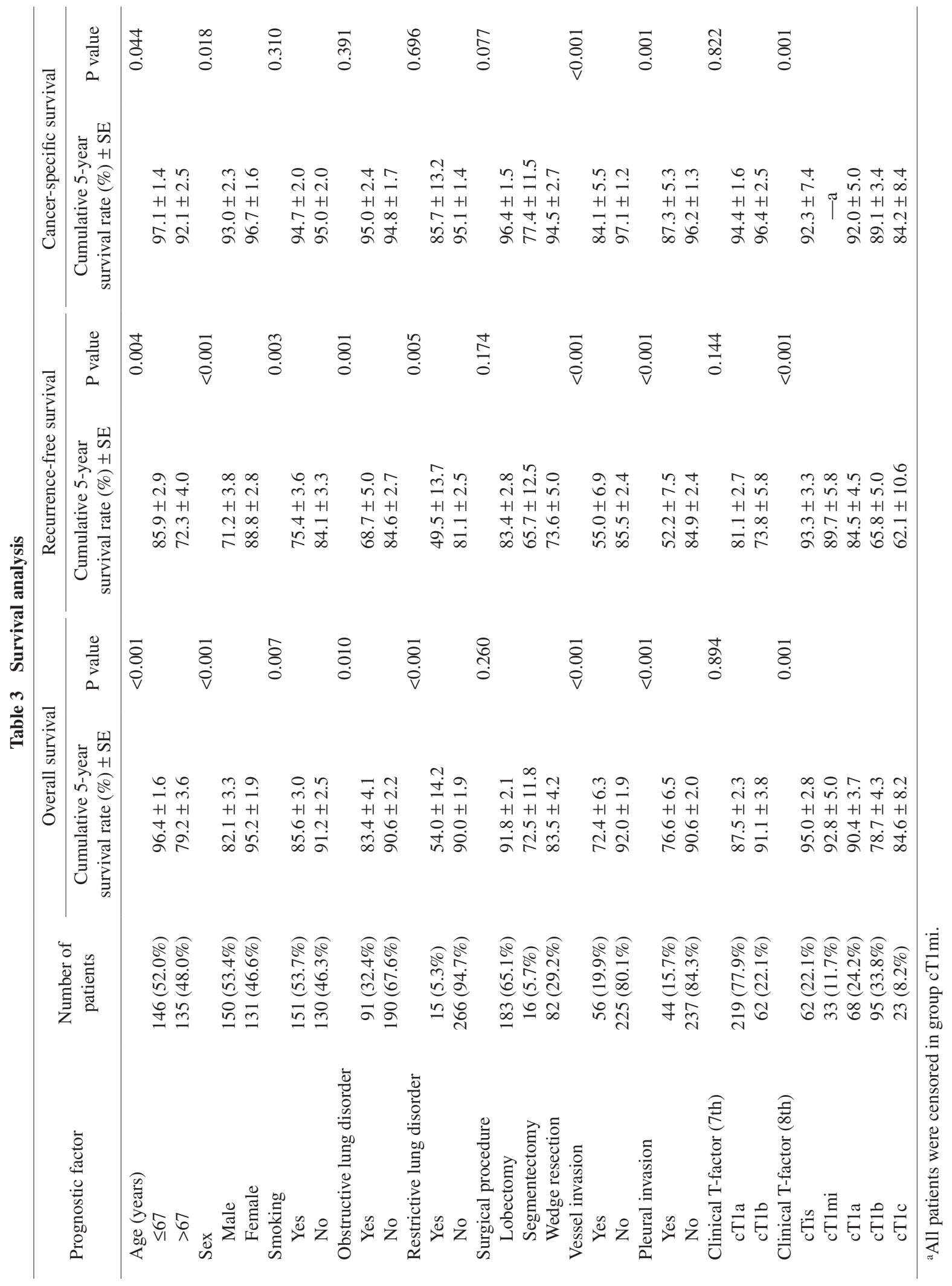



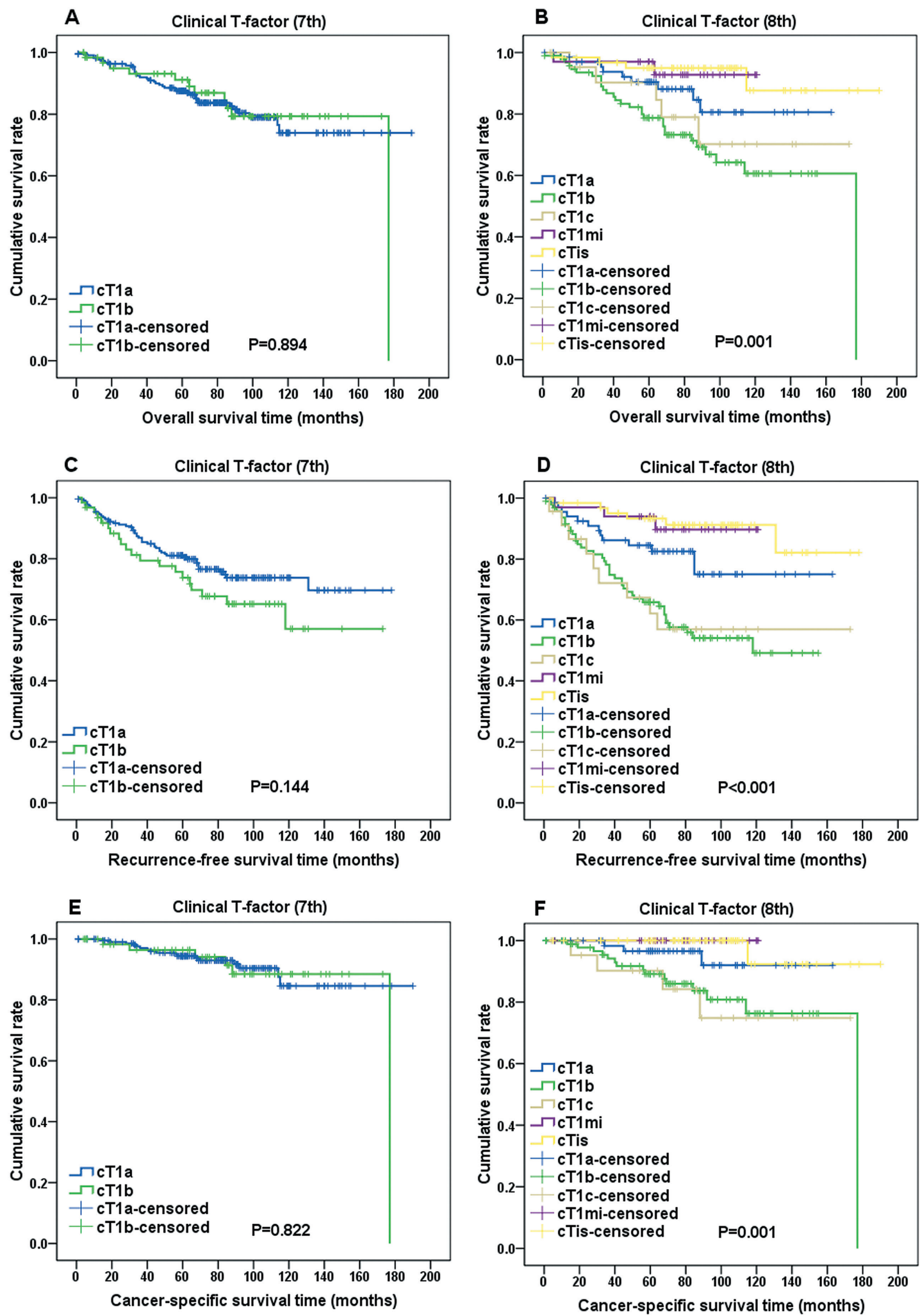

Fig. 1 Comparison of clinical T-factors between the 7th and 8th editions of the UICC-TNM classification by survival analysis. The overall survival curve of patients stratified with clinical T-factors (8th) (B) was more clearly separated than that of the 7th $(\mathbf{A})(\mathrm{P}=0.001$ vs. $\mathrm{P}=0.894)$. The recurrence-free survival curve of patients stratified with clinical T-factors (8th) (D) was more clearly separated than that of the 7 th $(\mathbf{C})(\mathrm{P}<0.001$ vs. $\mathrm{P}=0.144)$. The cancerspecific survival curve of patients stratified with clinical T-factors $(8 \mathrm{th})(\mathbf{F})$ was more clearly separated than that of the 7th $(\mathbf{E})(\mathrm{P}=0.001$ vs. $\mathrm{P}=0.822)$. UICC: union for international cancer control; TNM: tumor node metastasis 
TNM classification, the clinical physicians just measured the whole size of GGOs on HRCT like other solid lung cancers. But recently, several papers concluded that the postoperative survival rate of lung adenocarcinomas depends on the size of the solid part, not of the whole tumor in GGNs. ${ }^{17}{ }^{18)}$ The newest 8 th edition of the UICCTNM classification for lung cancer has been effective since January 2017, it initiated a new method of measuring tumor size of GGN lung adenocarcinoma, instead of the whole tumor size, the solid components size was used as the clinical T descriptor. ${ }^{19)}$ However, the 8th edition of the UICC-TNM classification was based on a worldwide database in which the size of the tumor was not defined as that of solid part. And recently, a research concluded that the whole size, not the solid part size of GGN lung adenocarcinoma was the independent prognostic factor for overall and disease-free survival. ${ }^{20)}$

Then here comes the questions: Is the new measuring method really suitable for the GGN lung adenocarcinoma? And how is the accuracy? We can answer the questions by comparing the prognostic significance of clinical T-factors between the 7th and the 8th editions, for they represent the old and the new measuring methods, respectively. And the results of this study showed that the clinical T-factors (8th) are more statistically significant than that of the 7th in predicting the postoperative prognosis of the patients in this study (Fig. 1). In other words, the new measuring method for GGN lung adenocarcinoma adopted in the 8th edition of the UICCTNM classification is more accurate than the previous one in predicting the postoperative prognosis of patients with clinical stage 0-IA lung adenocarcinoma.

In our study, we compared the 8th and the 7th editions in three kinds of survivals: the overall survival, the recurrence-free survival, and the cancer-specific survival (Fig. 1). With the P values 0.001 vs. 0.894 in the overall survival, $<0.001$ vs. 0.144 in the recurrence-free survival, and 0.001 vs. 0.822 in the cancer-specific survival, respectively, it was proved that the 8th edition of the UICC-TNM classification predicts postoperative prognosis more precisely than the 7 th one in clinical stage 0-IA lung adenocarcinoma. The possible reasons are as follows: First of all, the stage distribution of the population, which included in the research project the 8th edition based on, has been changed. Early stages (stage I) lung cancer are predominant, especially in Asia and North America. ${ }^{4)}$ So it will be more accurate when using the 8th edition to evaluate the early stage (stage I) lung cancer data from Asia, and all the cases in this study are stage 0-IA from Japan. Besides, the staging criteria for GGN lung adenocarcinoma are supplemented in the 8th edition. So, it will also be more accurate when using the 8th edition to evaluate the data of GGN lung adenocarcinomas, and the pure GGN and part solid GGN lung adenocarcinomas have 171 of 281 cases $(60.85 \%)$ altogether in this study.

However, there are also limitations in the study. First, only clinical stage 0-IA lung adenocarcinoma is discussed. Second, the sample size is not big enough. Finally, the representation is poor for all the patients are from Japan. Further assessment in a larger multi-center study which covers pathological and more advanced stage will be necessary.

\section{Conclusion}

This study shows that the 8th edition of the UICCTNM classification predicts postoperative prognosis more precisely than the 7th one in clinical stage 0-IA lung adenocarcinoma. And the new measuring method for GGN lung adenocarcinoma adopted in the 8th edition of the UICC-TNM classification is more accurate than the previous one in predicting the postoperative prognosis of patients with clinical stage 0-IA lung adenocarcinoma.

\section{Disclosure Statement}

We have no conflicts of interest.

\section{References}

1) Torre LA, Siegel RL, Jemal A. Lung cancer statistics. Adv Exp Med Biol 2016; 893: 1-19.

2) Torre LA, Bray F, Siegel RL, et al. Global cancer statistics, 2012. CA Cancer J Clin 2015; 65: 87-108.

3) Sobin LH, Gospodarowicz MK, Wittekind Ch. TNM classification of malignant tumours. 7th edition. Chichester, West Sussex, UK; Hoboken, NJ: Wiley-Blackwell, 2010; pp 138-46.

4) Rami-Porta R, Bolejack V, Giroux DJ, et al. The IASLC lung cancer staging project: the new database to inform the eighth edition of the TNM classification of lung cancer. J Thorac Oncol 2014; 9: 1618-24.

5) Brierley J, Gospodarowicz MK, Wittekind Ch. TNM classification of malignant tumours. 8th edition. Chichester, West Sussex, UK; Hoboken, NJ: John Wiley \& Sons, Inc., 2017; pp 106-12.

6) Henschke CI, Yankelevitz DF, Mirtcheva R, et al. CT screening for lung cancer: frequency and significance of part-solid and nonsolid nodules. AJR Am J Roentgenol 2002; 178: 1053-7. 
7) Burdett S, Pignon JP, Tierney J, et al. Adjuvant chemotherapy for resected early-stage non-small cell lung cancer. Cochrane Database Syst Rev 2015; doi: 10.1002/14651858.CD011430.

8) NSCLC Meta-analysis Collaborative Group. Preoperative chemotherapy for non-small-cell lung cancer: a systematic review and meta-analysis of individual participant data. Lancet 2014; 383: 1561-71.

9) Frazier HA, Robertson JE, Dodge RK, et al. The value of pathologic factors in predicting cancer-specific survival among patients treated with radical cystectomy for transitional cell carcinoma of the bladder and prostate. Cancer 1993; 71: 3993-4001.

10) Jang HJ, Lee KS, Kwon OJ, et al. Bronchioloalveolar carcinoma: focal area of ground-glass attenuation at thin-section CT as an early sign. Radiology 1996; 199: 485-8.

11) Okada M, Nishio W, Sakamoto T, et al. Correlation between computed tomographic findings, bronchioloalveolar carcinoma component, and biologic behavior of small-sized lung adenocarcinomas. J Thorac Cardiovasc Surg 2004; 127: 857-61.

12) Yang ZG, Sone $S$, Takashima $S$, et al. High-resolution CT analysis of small peripheral lung adenocarcinomas revealed on screening helical CT. AJR Am J Roentgenol 2001; 176: 1399-407.

13) Travis WD, Garg K, Franklin WA, et al. Evolving concepts in the pathology and computed tomography imaging of lung adenocarcinoma and bronchioloalveolar carcinoma. J Clin Oncol 2005; 23: 3279-87.
14) Travis WD, Brambilla E, Burke A, et al. WHO classification of tumours of the lung, pleura, thymus and heart. Lyon: IARC Press, 2015; pp 34-48.

15) Carter D, Vazquez M, Flieder DB, et al. Comparison of pathologic findings of baseline and annual repeat cancers diagnosed on CT screening. Lung Cancer 2007; 56: 193-9.

16) Park CM, Goo JM, Lee HJ, et al. Nodular groundglass opacity at thin-section CT: histologic correlation and evaluation of change at follow-up. Radiographics 2007; 27: 391-408.

17) Hwang EJ, Park CM, Ryu Y, et al. Pulmonary adenocarcinomas appearing as part-solid ground-glass nodules: is measuring solid component size a better prognostic indicator? Eur Radiol 2015; 25: 558-67.

18) Tsutani Y, Miyata Y, Nakayama H, et al. Prognostic significance of using solid versus whole tumor size on high-resolution computed tomography for predicting pathologic malignant grade of tumors in clinical stage IA lung adenocarcinoma: a multicenter study. J Thorac Cardiovasc Surg 2012; 143: 607-12.

19) Travis WD, Asamura H, Bankier AA, et al. The IASLC lung cancer staging project: proposals for coding $\mathrm{T}$ categories for subsolid nodules and assessment of tumor size in part-solid tumors in the forthcoming eighth edition of the TNM classification of lung cancer. J Thorac Oncol 2016; 11: 1204-23.

20) Song SH, Ahn JH, Lee HY, et al. Prognostic impact of nomogram based on whole tumour size, tumour disappearance ratio on CT and SUVmax on PET in lung adenocarcinoma. Eur Radiol 2016; 26: 1538-46. 